\title{
Nonlinear trident pair production in an arbitrary plane wave: A focus on the properties of the transition amplitude
}

\author{
F. Mackenroth ${ }^{1, *}$ and A. Di Piazza ${ }^{2, \dagger}$ \\ ${ }^{1}$ Max Planck Institute for the Physics of Complex Systems, \\ Nöthnitzer Straße 38, D-01187 Dresden, Germany \\ ${ }^{2}$ Max Planck Institute for Nuclear Physics, Saupfercheckweg 1, D-69117 Heidelberg, Germany
}

(Received 4 May 2018; published 11 December 2018)

\begin{abstract}
The process of nonlinear electron-positron pair production by an electron colliding with an arbitrary plane-wave electromagnetic field (nonlinear trident pair production) is studied analytically and numerically. Special emphasis is put on the properties of the transition amplitude. In fact, its original expression as resulting from applying the Wick's theorem turns out to be divergent. By utilizing a functional relation derived from gauge invariance, however, the amplitude is regularized and investigated in different regimes. In particular, the amplitude is divided into a two-step and a one-step contribution, depending on the scaling dependence on the laser pulse duration. The corresponding contributions to the positron angular distribution spectra and the resulting interference terms are studied numerically, emphasizing the possibility of measuring experimentally the one-step contribution.
\end{abstract}

DOI: 10.1103/PhysRevD.98.116002

\section{INTRODUCTION}

The prediction of QED that massive particle-antiparticle pairs can be created solely from electromagnetic fields is certainly among its prominent features. The recent progress in laser technology (see, e.g., [1-4]) opens the possibility of observing the production of electron-positron pairs in the collision of high-energy photons and intense laser beams [nonlinear Breit-Wheeler pair production (NBWPP)]. This process has been thoroughly investigated theoretically in recent years, also accounting for effects of the laser pulse form [5-11] (see [12] for publications until 2012). Since usually high-energy photons are produced via electron backscattering, alternatively, an electron-positron pair can be produced inside a strong laser field by a high-energy photon emitted by an ultrarelativistic electron colliding with the same laser field [nonlinear trident pair production (NTPP)]. Conventionally, the process can be described as commencing via two channels where loosely speaking the photon emission and pair production either occur at the same laser phase (direct channel) or at two separate ones (cascade channel). A unified theoretical description based on strong-field QED has been recently proposed to analyze

\footnotetext{
*mafelix@pks.mpg.de

dipiazza@mpi-hd.mpg.de
}

Published by the American Physical Society under the terms of the Creative Commons Attribution 4.0 International license. Further distribution of this work must maintain attribution to the author(s) and the published article's title, journal citation, and DOI. Funded by SCOAP ${ }^{3}$.
NTPP in a plane wave $[13,14]$ and in a constant-crossed field [15].

For an electron (mass $m$ and charge $e<0$ ) of initial fourmomentum $p_{i}^{\mu}=\left(\varepsilon_{i}, \boldsymbol{p}_{i}\right)$, with $\varepsilon_{i}=\sqrt{m^{2}+\boldsymbol{p}_{i}^{2}}$ (units with $\hbar=c=4 \pi \epsilon_{0}=1$ are used throughout), colliding with a plane wave of central angular frequency $\omega_{0}$, electric field amplitude $E$ and central wave four-vector $k_{0}^{\mu}=\left(\omega_{0}, \boldsymbol{k}_{0}\right)$, the total NTPP probability is controlled by the two Lorentz- and gauge-invariant parameters $\xi=|e| E / m \omega_{0}$ and $\chi=\left(\left(k_{0} p_{i}\right) / m^{2}\right) E / E_{\mathrm{cr}}$, where $E_{\mathrm{cr}}=m^{2} /|e|$ is the critical field of QED [16] and we introduced the short notation $(a b)=a_{\mu} b^{\mu}$ [the metric tensor is $\eta^{\mu \nu}=$ $\operatorname{diag}(+1,-1,-1,-1)]$. An exact inclusion of the laser field in the calculations is necessary for $\xi \gtrsim 1[17,18]$, which is nowadays routinely achieved at optical laser facilities [2]. At $\xi \gg 1$ NTPP occurs with the absorption of a large number of laser photons and is essentially controlled only by the parameter $\chi$ : it is exponentially suppressed for $\chi \ll 1$ and becomes sizable at $\chi \gtrsim 1$. Present day technology allows for optical lasers with $\xi \sim 10^{2}$ [1] and for electron beams with $\varepsilon_{i} \approx 4 \mathrm{GeV}$ produced via laser wakefield acceleration [19], allowing for a thorough experimental investigation of NTPP also within all-optical setups.

So far only one experiment on NTPP has been successfully carried out [20,21], where, however, the direct channel was strongly suppressed. For some time quantitative theoretical studies of NTPP were available only in the idealized cases of monochromatic lasers [13] and constantcrossed fields [15]. The latter study particularly assessed the validity of approximating trident pair production as a sequence of photon emission and Breit-Wheeler pair 
production, i.e., neglecting the direct channel, in a constantcrossed field background, as is commonly done in numerical simulations of laser-plasma interactions [22-24], and neglected the interference between exchange diagrams. The possibility of suppressing the cascade channel has also been discussed in [13] in the perturbative regime $\xi \ll 1$ at $\omega_{0} \sim 10 \mathrm{eV}$. Relying on the laser field being (almost) monochromatic, in that regime the direct channel is found to be either dominating or comparable with respect to the cascade channel for different laser frequencies. Most recently, however, studies of NTPP were amended to also include the exchange diagram interference in an arbitrary plane-wave field [25] as well as in a constant-crossed field [26], finding it to further suppress the contribution of the direct channel for small quantum parameters $\chi$. Additionally, the former investigation revealed that for large $\xi$, the direct contribution and the interference between the direct and cascade amplitudes are negligible [25], further supporting the basic assumption underlying numerical particle-in-cell schemes to approximate NTPP as the product of the probabilities for photon emission and pair production. Previous studies had mostly focused on the cascade contribution, which was obtained by employing the optical theorem and the two-loop mass operator [27-30].

The present work focuses on formal aspects of the full NTPP amplitude in arbitrary plane-wave fields, different from earlier studies. We put forward a possible scheme to analytically disentangle the amplitude of NTPP in an arbitrary plane-wave field into direct and cascade channels putting particular emphasis on the amplitude's gauge invariance. We demonstrate how this disentanglement can be employed to identify an explicit cascade contribution, facilitating identification of the remaining parts as a true second-order noncascade contribution. We also show how at the amplitude level this split-up naturally yields a phase-ordered cascade and a direct contribution depending only on one laser phase variable. Concerning the experimental observability, we indicate by means of numerical simulations how in ultrashort laser pulses with $\xi \gg 1$ the two channels scale in the energy distribution of the produced positron and one of the electrons.

\section{THE TRANSITION AMPLITUDE}

The background plane-wave field is described by the four-vector potential $A^{\mu}(\phi)$, which only depends on the light-cone time $\phi=t-\boldsymbol{n} \cdot \boldsymbol{x}$. Here, the unit vector $\boldsymbol{n}$ indicates the propagation direction of the plane wave. By introducing the four-dimensional quantity $n^{\mu}=(1, \boldsymbol{n})$ and by recalling that the metric tensor reads $\eta^{\mu \nu}=$ $\operatorname{diag}(+1,-1,-1,-1)$, it is $\phi=(n x)$. Having in mind obvious differential properties of the four-vector potential $A^{\mu}(\phi)$ and its derivatives, it is clear that $A^{\mu}(\phi)$ is a solution of the free Maxwell's equation $\square A^{\mu}=0$, with $\square=\partial_{\nu} \partial^{\nu}$. We also assume to work in the Lorenz gauge $\partial_{\mu} A^{\mu}=0$, with the additional constraint $A^{0}(\phi)=0$. Thus, if we represent
$A^{\mu}(\phi)$ in the form $A^{\mu}(\phi)=(0, A(\phi))$, then the Lorenzgauge condition implies $\boldsymbol{n} \cdot \boldsymbol{A}^{\prime}(\phi)=0$, with the prime indicating the derivative with respect to $\phi$. If we make the additional physically reasonable assumption that $\lim _{\phi \rightarrow \pm \infty} \boldsymbol{A}(\phi)=0$, it results that $\boldsymbol{n} \cdot \boldsymbol{A}(\phi)=0$. By also introducing two four-vectors $a_{j}^{\mu}=\left(0, \boldsymbol{a}_{j}\right)$, with $j=1,2$, such that $\left(n a_{j}\right)=-\boldsymbol{n} \cdot \boldsymbol{a}_{j}=0$ and $\left(a_{i} a_{j}\right)=-\boldsymbol{a}_{i} \cdot \boldsymbol{a}_{j}=-\delta_{i j}$, the most general form of the vector potential $\boldsymbol{A}(\phi)$ reads $\boldsymbol{A}(\phi)=\psi_{1}(\phi) \boldsymbol{a}_{1}+\psi_{2}(\phi) \boldsymbol{a}_{2}$, where the two functions $\psi_{j}(\phi)$ are arbitrary provided that $\lim _{\phi \rightarrow \pm \infty} \psi_{j}(\phi)=0$ and that they are analytically sufficiently well behaved. By introducing $\tilde{n}^{\mu}=(1,-\boldsymbol{n}) / 2$, it is clear that the four-dimensional quantities $n^{\mu}, \tilde{n}^{\mu}$, and $a_{j}^{\mu}$ fulfill the completeness relation: $\eta^{\mu \nu}=n^{\mu} \tilde{n}^{\nu}+\tilde{n}^{\mu} n^{\nu}-a_{1}^{\mu} a_{1}^{\nu}-a_{2}^{\mu} a_{2}^{\nu} \quad$ [note that $(n \tilde{n})=1$ and $\left(\tilde{n} a_{j}\right)=0$ ]. Below, we will refer to the longitudinal $(n)$ direction as the direction along the unit vector $\boldsymbol{n}$ and to the transverse $(\perp)$ plane as the plane spanned by the two perpendicular unit vectors $\boldsymbol{a}_{j}$. In this respect, together with the light-cone time $\phi=t-x_{n}$, with $x_{n}=\boldsymbol{n} \cdot \boldsymbol{x}$, we also introduce the remaining three light-cone coordinates $T=(\tilde{n} x)=\left(t+x_{n}\right) / 2$, and $\boldsymbol{x}_{\perp}=\left(x_{a_{1}}, x_{a_{2}}\right)=$ $\left(\boldsymbol{x} \cdot \boldsymbol{a}_{1}, \boldsymbol{x} \cdot \boldsymbol{a}_{2}\right)$. Analogously, the light-cone coordinates of an arbitrary four-vector $v^{\mu}=\left(v^{0}, \boldsymbol{v}\right)$ will be indicated as $v_{-}=(n v)=v^{0}-v_{n}$, with $v_{n}=\boldsymbol{n} \cdot \boldsymbol{v}, v_{+}=(\tilde{n} v)=\left(v^{0}+v_{n}\right) / 2$, and $\boldsymbol{v}_{\perp}=\left(v_{a_{1}}, v_{a_{2}}\right)=\left(\boldsymbol{v} \cdot \boldsymbol{a}_{1}, \boldsymbol{v} \cdot \boldsymbol{a}_{2}\right)$.

Now, the amplitude $S_{f i}$ of nonlinear trident pair production in the Furry picture at the leading order is given by (see Fig. 1 for the corresponding Feynman diagrams)

$$
\begin{aligned}
S_{f i}= & i e^{2} \int d^{4} x d^{4} y \bar{U}_{p_{e}, s_{e}}(y) \gamma^{\mu} V_{p_{p}, s_{p}}(y) D_{\mu \nu}(y-x) \\
& \times \bar{U}_{p_{f}, s_{f}}(x) \gamma^{\nu} U_{p_{i}, s_{i}}(x)-\{f \leftrightarrow e\},
\end{aligned}
$$

where $\gamma^{\mu}$ are the Dirac gamma matrices and

$$
U_{p, s}(x)=\left[1+\frac{e \hat{n} \hat{A}(\phi)}{2 p_{-}}\right] e^{i\left\{-(p x)-\int_{0}^{\phi} d \varphi\left[\frac{e(p A(\varphi))}{p-} \frac{e^{2} A^{2}(\varphi)}{2 p-}\right]\right\}} \frac{u_{p, s}}{\sqrt{2 \varepsilon}},
$$

$$
V_{p, s}(x)=\left[1-\frac{e \hat{n} \hat{A}(\phi)}{2 p_{-}}\right] e^{i\left\{(p x)-\int_{0}^{\phi} d \varphi\left[\frac{e(p A(\varphi))}{p_{-}}+\frac{e^{A^{2} A^{2}(\varphi)}}{2 p-}\right]\right\}} \frac{v_{p, s}}{\sqrt{2 \varepsilon}}
$$

are the positive- and negative-energy Volkov states with on-shell four-momentum $p^{\mu}=(\varepsilon, \boldsymbol{p})$ and spin quantum number $s[18]$, where

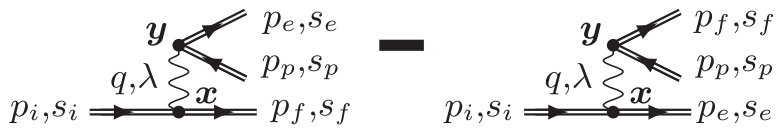

FIG. 1. Lowest-order Feynman diagrams of NTPP. 


$$
D^{\mu \nu}(y-x)=\int \frac{d^{4} q}{(2 \pi)^{4}} \frac{4 \pi}{q^{2}+i 0} \eta^{\mu \nu} e^{-i q(y-x)}
$$

is the photon propagator, and where the symbol $\{f \leftrightarrow e\}$ indicates that the expression on its left has to be subtracted with the quantum numbers $p_{f}, s_{f}$ and $p_{e}, s_{e}$ exchanged according to the Fermi-Dirac statistics. We stress here that in contrast to [25] most of our analytical derivations are based on Eq. (1), i.e., on the process amplitude rather than its rate. In the above expression of the Volkov states, we have assumed a unit quantization volume, we have introduced the notation $\hat{v}=\gamma^{\mu} v_{\mu}$ for an arbitrary fourdimensional quantity $v_{\mu}$ and the free bispinors $u_{p, s}$ and $v_{p s}$, which are solutions of the equations $(\hat{p}-m) u_{p, s}=0$ and $(\hat{p}+m) v_{p, s}=0$, respectively [18].

By indicating with an index $x$ and $y$ the light-cone coordinates corresponding to each vertex, due to the symmetry of the plane wave, it is possible to carry out the six integrations on the transverse coordinates and on $T_{x}$ and $T_{y}$, such that the amplitude $S_{f i}$ has the form

$$
\begin{aligned}
S_{f i}= & \frac{i e^{2}}{k_{-}} \frac{2 \pi}{\sqrt{16 \varepsilon_{i} \varepsilon_{f} \varepsilon_{e} \varepsilon_{p}}}(2 \pi)^{3} \delta\left(p_{e,-}+p_{f,-}+p_{p,-}-p_{i,-}\right) \delta^{(2)}\left(\boldsymbol{p}_{e, \perp}+\boldsymbol{p}_{f, \perp}+\boldsymbol{p}_{p, \perp}-\boldsymbol{p}_{i, \perp}\right) \\
& \times \int d \phi_{x} d \phi_{y} \int \frac{d q_{+}}{2 \pi} e^{-i\left[S_{p_{e}}\left(\phi_{y}\right)-S_{-p_{p}}\left(\phi_{y}\right)+S_{p_{f}}\left(\phi_{x}\right)-S_{p_{i}}\left(\phi_{x}\right)\right]} \frac{e^{-i q_{+}\left(\phi_{y}-\phi_{x}\right)}}{q_{+}-\frac{\boldsymbol{k}_{\perp}^{2}}{2 k_{-}}+i 0} \\
& \times \bar{u}_{e}\left[1-\frac{e \hat{n} \hat{A}\left(\phi_{y}\right)}{2 p_{e,-}}\right] \gamma^{\mu}\left[1-\frac{e \hat{n} \hat{A}\left(\phi_{y}\right)}{2 p_{p,-}}\right] v_{p} \bar{u}_{f}\left[1-\frac{e \hat{n} \hat{A}\left(\phi_{x}\right)}{2 p_{f,-}}\right] \gamma_{\mu}\left[1+\frac{e \hat{n} \hat{A}\left(\phi_{x}\right)}{2 p_{i,-}}\right] u_{i}-\{f \leftrightarrow e\},
\end{aligned}
$$

where

$$
S_{p}(\phi)=-p_{+} \phi-\int_{0}^{\phi} d \varphi\left[\frac{e(p A(\varphi))}{p_{-}}-\frac{e^{2} A^{2}(\varphi)}{2 p_{-}}\right]
$$

where the single index in the bispinors indicates both the corresponding four-momentum and the spin quantum numbers, and where $k_{-}=p_{i,-}-p_{f,-}=p_{e,-}+p_{p,-}$ and $\boldsymbol{k}_{\perp}=\boldsymbol{p}_{i, \perp}-\boldsymbol{p}_{f, \perp}=\boldsymbol{p}_{e, \perp}+\boldsymbol{p}_{p, \perp}$. The integral in $q_{+}$can also be easily taken by means of the residue method and the amplitude becomes

$$
\begin{aligned}
S_{f i}= & \frac{e^{2}}{\sqrt{16 \varepsilon_{i} \varepsilon_{f} \varepsilon_{e} \varepsilon_{p}}} \frac{2 \pi}{k_{-}}(2 \pi)^{3} \delta\left(p_{e,-}+p_{f,-}+p_{p,-}-p_{i,-}\right) \delta^{(2)}\left(\boldsymbol{p}_{e, \perp}+\boldsymbol{p}_{f, \perp}+\boldsymbol{p}_{p, \perp}-\boldsymbol{p}_{i, \perp}\right) \\
& \times \int d \phi_{x} d \phi_{y} \theta\left(\phi_{y}-\phi_{x}\right) \bar{u}_{e}\left[1-\frac{e \hat{n} \hat{A}\left(\phi_{y}\right)}{2 p_{e,-}}\right] \gamma^{\mu}\left[1-\frac{e \hat{n} \hat{A}\left(\phi_{y}\right)}{2 p_{p,-}}\right] v_{p} \\
& \times \bar{u}_{f}\left[1-\frac{e \hat{n} \hat{A}\left(\phi_{x}\right)}{2 p_{f,-}}\right] \gamma_{\mu}\left[1+\frac{e \hat{n} \hat{A}\left(\phi_{x}\right)}{2 p_{i,-}}\right] u_{i} e^{-i\left[S_{\mathrm{BW}}\left(\phi_{y}\right)+S_{C}\left(\phi_{x}\right)\right]}-\{f \leftrightarrow e\},
\end{aligned}
$$

where $\theta(\cdot)$ is the step function and

$$
\begin{aligned}
S_{C}(\phi) & =-k_{+} \phi+S_{p_{f}}(\phi)-S_{p_{i}}(\phi) \\
& =\int_{0}^{\phi} d \varphi\left[p_{i,+}-p_{f,+}-k_{+}+\frac{e\left(p_{i} A\right)}{p_{i,-}}-\frac{e\left(p_{f} A\right)}{p_{f,-}}-\frac{e^{2} A^{2}}{2 p_{i,-}}+\frac{e^{2} A^{2}}{2 p_{f,-}}\right] \\
S_{\mathrm{BW}}(\phi) & =k_{+} \phi+S_{p_{e}}(\phi)-S_{-p_{p}}(\phi) \\
& =\int_{0}^{\phi} d \varphi\left[k_{+}-p_{e,+}-p_{p,+}+\frac{e\left(p_{p} A\right)}{p_{p,-}}-\frac{e\left(p_{e} A\right)}{p_{e,-}}+\frac{e^{2} A^{2}}{2 p_{p,-}}+\frac{e^{2} A^{2}}{2 p_{e,-}}\right]
\end{aligned}
$$

are the corresponding phases of nonlinear Compton scattering and of nonlinear Breit-Wheeler pair production, respectively. It is worth noticing that, after performing the integral in $q_{+}$, the four-momentum of the intermediate photon appears as being on shell, i.e., $k_{+}=\boldsymbol{k}_{\perp}^{2} / 2 k_{-}$. Below, we will only consider the experimentally most relevant case of a linearly polarized plane wave. Thus, we write the four-vector potential as $A^{\mu}(\phi)=A_{0}^{\mu} \psi(\phi)$, such that $S_{C}(\phi)=\int_{0}^{\phi} d \varphi\left[\alpha_{C} \psi(\varphi)+\beta_{C} \psi^{2}(\varphi)+\gamma_{C}\right]$ and $S_{\mathrm{BW}}(\phi)=\int_{0}^{\phi} d \varphi\left[\alpha_{\mathrm{BW}} \psi(\varphi)+\beta_{\mathrm{BW}} \psi^{2}(\varphi)+\gamma_{\mathrm{BW}}\right]$, with 


$$
\begin{array}{cc}
\alpha_{C}=\frac{e\left(p_{i} A_{0}\right)}{p_{i,-}}-\frac{e\left(p_{f} A_{0}\right)}{p_{f,-}}, \quad \alpha_{\mathrm{BW}}=\frac{e\left(p_{p} A_{0}\right)}{p_{p,-}}-\frac{e\left(p_{e} A_{0}\right)}{p_{e,-}}, \\
\beta_{C}=\frac{e^{2} A_{0}^{2}}{2} \frac{k_{-}}{p_{i,-} p_{f,-}}, \quad \beta_{\mathrm{BW}}=\frac{e^{2} A_{0}^{2}}{2} \frac{k_{-}}{p_{e,-} p_{p,-}}, \\
\gamma_{C}=p_{i,+}-p_{f,+}-k_{+}, \quad \gamma_{\mathrm{BW}}=k_{+}-p_{e,+}-p_{p,+} .
\end{array}
$$

From the expression of the amplitude $S_{f i}$ in Eq. (7) one could think that the whole amplitude only contains a contribution to the two-step channel or cascade process, as it contains a phase-ordered double integral of the "product" of the nonlinear Compton scattering amplitude and of the nonlinear Breit-Wheeler pair production amplitudes, both with the photon being on shell. This is, however, not the case mainly because the amplitude $S_{f i}$ contains contributions from all polarizations of the intermediate photon, whereas the cascade process only stems from photons having transverse polarization. Another reason is that the amplitude in Eq. (7) is, on the one hand, still divergent because some terms do not contain the external field in the preexponential function which, on the other hand, can be regularized by enforcing gauge invariance. The second reason, though, is less fundamental in the sense that, in the case of a constant-crossed field, it does not play a role because also the phase integrals which do not contain the external field in the preexponential function do converge. In the present case, precisely the phase integrals corresponding to these terms diverge.

First, we impose that the amplitude $S_{f i}$ is manifestly gauge invariant by going back to the expression in Eq. (5) and requiring that the amplitude not change if the tensor $\eta^{\mu \nu}$ in the photon propagator is replaced by $\eta^{\mu \nu}+q^{\mu} \lambda^{\nu}(q)+q^{\nu} \lambda^{\mu}(q)$, where $\lambda^{\mu}(q)$ is an arbitrary function of $q^{\mu}$. It is clear from the appearance of the energy-momentum conserving delta-functions that we can already assume here that $q_{-}=k_{-}$and $\boldsymbol{q}_{\perp}=$ $\boldsymbol{k}_{\perp}$ from the beginning. Moreover, in order to obtain all the required regularization conditions for the integrals, it is sufficient to assume that $\lambda^{\mu}(q)$ is a constant four-vector. Then, since QED is gauge invariant, once the integrals are convergent, we can be confident that the resulting amplitude is invariant under a more general transformation of $\eta^{\mu \nu}$ than the one studied above. Now, one can easily show that by exploiting the energy-momentum conservation laws $p_{i}^{\mu}+$ $\left(k_{+}+p_{f,+}-p_{i,+}\right) n^{\mu}=p_{f}^{\mu}+k^{\mu}$ and $k^{\mu}+\left(p_{e,+}+p_{p,+}-k_{+}\right) n^{\mu}=$ $p_{e}^{\mu}+p_{p}^{\mu}$, the invariance of the amplitude under the mentioned gauge transformation is guaranteed if the "regularizing" conditions

$$
\begin{gathered}
\int d \phi \psi^{a}(\phi) e^{-i\left[S_{C}(\phi)+S_{\mathrm{BW}}(\phi)\right]}=-i \int d \phi_{x} d \phi_{y} \theta\left(\phi_{y}-\phi_{x}\right)\left[\alpha_{C} \psi\left(\phi_{x}\right)+\beta_{C} \psi^{2}\left(\phi_{x}\right)+\gamma_{C}\right] \psi^{a}\left(\phi_{y}\right) e^{-i\left[S_{C}\left(\phi_{x}\right)+S_{\mathrm{BW}}\left(\phi_{y}\right)\right]}, \\
\int d \phi \psi^{b}(\phi) e^{-i\left[S_{C}(\phi)+S_{\mathrm{BW}}(\phi)\right]}=i \int d \phi_{x} d \phi_{y} \theta\left(\phi_{y}-\phi_{x}\right)\left[\alpha_{\mathrm{BW}} \psi\left(\phi_{y}\right)+\beta_{\mathrm{BW}} \psi^{2}\left(\phi_{y}\right)+\gamma_{\mathrm{BW}}\right] \psi^{b}\left(\phi_{x}\right) e^{-i\left[S_{C}\left(\phi_{x}\right)+S_{\mathrm{BW}}\left(\phi_{y}\right)\right]},
\end{gathered}
$$

with $a, b=0,1,2$, are fulfilled. The connection between these integral relations and gauge invariance was previously pointed out for trident pair production [14] and two-photon emission [31,32]. By introducing the quantities

$$
\begin{gathered}
f_{a}=\int d \phi \psi^{a}(\phi) e^{-i\left[S_{C}(\phi)+S_{\mathrm{BW}}(\phi)\right]}, \\
f_{a b}=\int d \phi_{x} d \phi_{y} \theta\left(\phi_{y}-\phi_{x}\right) \psi^{a}\left(\phi_{x}\right) \psi^{b}\left(\phi_{y}\right) e^{-i\left[S_{C}\left(\phi_{x}\right)+S_{\mathrm{BW}}\left(\phi_{y}\right)\right]},
\end{gathered}
$$

it is clear that, among all of them, only $f_{0}, f_{00}, f_{01}, f_{10}, f_{02}$, and $f_{20}$ need to be regularized. The conditions in Eqs. (13) and (14) already guarantee that

$$
\begin{gathered}
f_{0 j}=\frac{1}{\gamma_{C}}\left(i f_{j}-\alpha_{C} f_{1 j}-\beta_{C} f_{2 j}\right), \\
f_{j 0}=-\frac{1}{\gamma_{\mathrm{BW}}}\left(i f_{j}+\alpha_{\mathrm{BW}} f_{j 1}+\beta_{\mathrm{BW}} f_{j 2}\right),
\end{gathered}
$$

with $j=1$, 2. By subtracting now Eqs. (13) and (14), we obtain that $f_{00}=-\left(\alpha_{C} f_{10}+\beta_{C} f_{20}+\alpha_{\mathrm{BW}} f_{01}+\beta_{\mathrm{BW}} f_{02}\right) /$ $\left(\gamma_{C}+\gamma_{\mathrm{BW}}\right)$ and, by exploiting the above regularizing relations for $f_{0 j}$ and $f_{j 0}$, that

$f_{00}=\frac{1}{\gamma_{C} \gamma_{\mathrm{BW}}}\left[i \frac{\alpha_{C} \gamma_{C}-\alpha_{\mathrm{BW}} \gamma_{\mathrm{BW}}}{\gamma_{C}+\gamma_{\mathrm{BW}}} f_{1}+i \frac{\beta_{C} \gamma_{C}-\beta_{\mathrm{BW}} \gamma_{\mathrm{BW}}}{\gamma_{C}+\gamma_{\mathrm{BW}}} f_{2}+\alpha_{C} \alpha_{\mathrm{BW}} f_{11}+\beta_{C} \beta_{\mathrm{BW}} f_{22}+\alpha_{C} \beta_{\mathrm{BW}} f_{12}+\alpha_{\mathrm{BW}} \beta_{C} f_{21}\right]$. 
Finally, the regularization condition for $f_{0}$ is obtained by summing Eqs. (13) and (14) as we obtain $f_{0}=$ $-(i / 2)\left[\left(\gamma_{C}-\gamma_{\mathrm{BW}}\right) f_{00}+\alpha_{C} f_{10}-\alpha_{\mathrm{BW}} f_{01}+\beta_{C} f_{20}-\beta_{\mathrm{BW}} f_{02}\right]$ and then, after some algebra,

$$
f_{0}=-\frac{1}{\gamma_{C}+\gamma_{\mathrm{BW}}}\left[\left(\alpha_{C}+\alpha_{\mathrm{BW}}\right) f_{1}+\left(\beta_{C}+\beta_{\mathrm{BW}}\right) f_{2}\right] .
$$

Now that all integrals are regularized, we can appropriately replace the divergent integrals in Eq. (7) and the regularized, explicitly gauge-invariant amplitude reads

$$
\begin{aligned}
S_{f i}= & \frac{e^{2}}{\sqrt{16 \varepsilon_{i} \varepsilon_{f} \varepsilon_{e} \varepsilon_{p}}} \frac{2 \pi}{k_{-}}(2 \pi)^{3} \delta\left(p_{e,-}+p_{f,-}+p_{p,-}-p_{i,-}\right) \delta^{(2)}\left(\boldsymbol{p}_{e, \perp}+\boldsymbol{p}_{f, \perp}+\boldsymbol{p}_{p, \perp}-\boldsymbol{p}_{i, \perp}\right) \\
& \times\left\langlei \int d \phi e ^ { - i [ S _ { \mathrm { BW } } ( \phi ) + S _ { C } ( \phi ) ] } \left\{\frac{\bar{u}_{e} \gamma^{\mu} v_{p} \bar{u}_{f} \gamma_{\mu} u_{i}}{\gamma_{C} \gamma_{\mathrm{BW}}\left(\gamma_{C}+\gamma_{\mathrm{BW}}\right)}\left[\left(\alpha_{C} \gamma_{C}-\alpha_{\mathrm{BW}} \gamma_{\mathrm{BW}}\right) \psi(\phi)+\left(\beta_{C} \gamma_{C}-\beta_{\mathrm{BW}} \gamma_{\mathrm{BW}}\right) \psi^{2}(\phi)\right]\right.\right. \\
& \left.-\frac{\bar{u}_{f} \gamma_{\mu} u_{i}}{2 \gamma_{C}} \bar{u}_{e}\left[\frac{e \hat{n} \hat{A}(\phi)}{p_{e,-}} \gamma^{\mu}+\gamma^{\mu} \frac{e \hat{n} \hat{A}(\phi)}{p_{p,-}}-\frac{e^{2} A^{2}(\phi) \hat{n}}{p_{e,-} p_{p,-}} n^{\mu}\right] v_{p}+\frac{\bar{u}_{e} \gamma_{\mu} v_{p}}{2 \gamma_{\mathrm{BW}}} \bar{u}_{f}\left[\frac{e \hat{n} \hat{A}(\phi)}{p_{f,-}} \gamma^{\mu}-\gamma^{\mu} \frac{e \hat{n} \hat{A}(\phi)}{p_{i,-}}+\frac{e^{2} A^{2}(\phi) \hat{n}}{p_{f,-} p_{i,-}} n^{\mu}\right] u_{i}\right\} \\
& \left.+\int d \phi_{x} d \phi_{y} \theta\left(\phi_{y}-\phi_{x}\right) M_{\mathrm{BW}}^{\mu}\left(\phi_{y}\right) M_{C, \mu}\left(\phi_{x}\right)\right\rangle-\{f \leftrightarrow e\},
\end{aligned}
$$

where we have introduced the regularized integrands

$$
\begin{gathered}
M_{C}^{\mu}(\phi)=\bar{u}_{f}\left\{-\frac{\gamma^{\mu}}{\gamma_{C}}\left[\alpha_{C} \psi(\phi)+\beta_{C} \psi^{2}(\phi)\right]-\frac{e \hat{n} \hat{A}(\phi)}{2 p_{f,-}} \gamma^{\mu}+\gamma^{\mu} \frac{e \hat{n} \hat{A}(\phi)}{2 p_{i,-}}-\frac{e^{2} A^{2}(\phi) \hat{n}}{2 p_{f,-} p_{i,-}} n^{\mu}\right\} u_{i} e^{-i S_{C}(\phi),} \\
M_{\mathrm{BW}}^{\mu}(\phi)=\bar{u}_{e}\left\{-\frac{\gamma^{\mu}}{\gamma_{\mathrm{BW}}}\left[\alpha_{\mathrm{BW}} \psi(\phi)+\beta_{\mathrm{BW}} \psi^{2}(\phi)\right]-\frac{e \hat{n} \hat{A}(\phi)}{2 p_{e,-}} \gamma^{\mu}-\gamma^{\mu} \frac{e \hat{n} \hat{A}(\phi)}{2 p_{p,-}}+\frac{e^{2} A^{2}(\phi) \hat{n}}{2 p_{e,-} p_{p,-}} n^{\mu}\right\} v_{p} e^{-i S_{\mathrm{BW}}(\phi)}
\end{gathered}
$$

of the amplitudes of nonlinear Compton scattering and nonlinear Breit-Wheeler pair production, respectively [it is clear that the substitution $e \leftrightarrow f$ has to be carried out also inside these amplitudes in Eq. (21)]. The advantage of this full regularization already on amplitude level is that in the numerical evaluation no further singular phase integrals are encountered (see Sec. III). As a downside, on the other hand, the resulting probabilities do not have a Gaussian form with respect to the transverse momenta of the final particle, which, unlike in [25], prevents us from performing the corresponding integrals analytically. However, as a sanity check, we have explicitly proven that by expanding Eq. (21) with respect to the laser amplitude up to first order, the perturbative amplitude of the linear trident process is obtained, as it follows by applying the standard Feynman rules in vacuum QED. This check is particularly important because it complements the other check at large fields discussed below Eq. (43). The gauge-invariant expression (21) of the amplitude $S_{f i}$ is already close to the separation between the one-step (direct) channel and two-step (cascade) channel that we want to obtain. As we have already mentioned, we still need to isolate in the cascade amplitude only the contribution due to the two transverse polarizations of the intermediate photon. It is convenient to construct a light-cone basis with the lightlike quantities $k^{\mu}$ and $n^{\mu}$, and with the two transverse polarization four-vectors $\Lambda_{j}^{\mu}=\left(n^{\mu} a_{j}^{\nu}-n^{\nu} a_{j}^{\mu}\right) k_{\nu} / k_{-}$. In fact, all these quantities fulfill the completeness relation $\eta^{\mu \nu}=\left(n^{\mu} k^{\nu}+n^{\nu} k^{\mu}\right) / k_{-}-$ $\Lambda_{1}^{\mu} \Lambda_{1}^{\nu}-\Lambda_{2}^{\mu} \Lambda_{2}^{\nu}$ and we can replace this expression of $\eta^{\mu \nu}$ in all the Lorentz contractions in Eq. (21). It is noteworthy that employing this replacement for separating the process amplitude into a coherent and cascade contribution is different from both Eqs. (11) and (15) of [25]. We will demonstrate below, however, that in the physical limit of a constant-crossed field the two approaches reduce to the same expression, equivalent to the commonly used approximation of the trident probability being given by a polarization summed product of the Compton and Breit-Wheeler probabilities [15]. This equivalence was demonstrated in [25] by employing an expansion of the total process probability in orders of $\xi^{-1}$, indicating that the expressions can indeed be expected to agree only in the limit $\xi \rightarrow \infty$, corresponding to considering a constant-crossed field. For smaller $\xi$ the splitting derived from replacing $\eta^{\mu \nu}$ by the above basis set is not unique, analogously to the split-up proposed in [25] in this regime. On the other hand, we wish to stress that due to this ambiguity the difference in the chosen split-ups does not indicate different physics, as a physical split-up into separate channels is only possible on a probability level in the limit of vanishing formation length 
$(\xi \rightarrow \infty)$ of the two subprocesses. In the regime $\xi \sim 1$, on the other hand, any deviation of the total probability, obtained from squaring Eq. (24), from the constant-crossed field predictions are physical and one may chose a split-up depending on which aspects of the process one wishes to study, as long as the cascade channel reduces to the constantcrossed field results in the appropriate limit $\xi \rightarrow \infty$. The result of our approach is

$$
\begin{aligned}
S_{f i}= & \frac{e^{2}}{\sqrt{16 \varepsilon_{i} \varepsilon_{f} \varepsilon_{e} \varepsilon_{p}}} \frac{2 \pi}{k_{-}}(2 \pi)^{3} \delta\left(p_{e,-}+p_{f,-}+p_{p,-}-p_{i,-}\right) \delta^{(2)}\left(\boldsymbol{p}_{e, \perp}+\boldsymbol{p}_{f, \perp}+\boldsymbol{p}_{p, \perp}-\boldsymbol{p}_{i, \perp}\right) \\
& \times\left\langlei \int d \phi e ^ { - i [ S _ { \mathrm { BW } } ( \phi ) + S _ { C } ( \phi ) ] } \left\{\frac{2}{k_{-}} \frac{\bar{u}_{e} \hat{n} v_{p} \bar{u}_{f} \hat{n} u_{i}}{\gamma_{C}+\gamma_{\mathrm{BW}}}\left[\left(\alpha_{C}+\alpha_{\mathrm{BW}}\right) \psi(\phi)+\left(\beta_{C}+\beta_{\mathrm{BW}}\right) \psi^{2}(\phi)\right]\right.\right. \\
& -\sum_{j} \frac{\bar{u}_{e} \hat{\Lambda}_{j} v_{p} \bar{u}_{f} \hat{\Lambda}_{j} u_{i}}{\gamma_{C} \gamma_{\mathrm{BW}}\left(\gamma_{C}+\gamma_{\mathrm{BW}}\right)}\left[\left(\alpha_{C} \gamma_{C}-\alpha_{\mathrm{BW}} \gamma_{\mathrm{BW}}\right) \psi(\phi)+\left(\beta_{C} \gamma_{C}-\beta_{\mathrm{BW}} \gamma_{\mathrm{BW}}\right) \psi^{2}(\phi)\right] \\
& \left.+\sum_{j} \frac{\bar{u}_{f} \hat{\Lambda}_{j} u_{i}}{2 \gamma_{C}} \bar{u}_{e}\left[\frac{e \hat{n} \hat{A}(\phi) \hat{\Lambda}_{j}}{p_{e,-}}+\frac{e \hat{\Lambda}_{j} \hat{n} \hat{A}(\phi)}{p_{p,-}}\right] v_{p}-\frac{\bar{u}_{e} \hat{\Lambda}_{j} v_{p}}{2 \gamma_{\mathrm{BW}}} \bar{u}_{f}\left[\frac{e \hat{n} \hat{A}(\phi) \hat{\Lambda}_{j}}{p_{f,-}}-\frac{e \hat{\Lambda} \hat{n} \hat{n}(\phi)}{p_{i,-}}\right] u_{i}\right\} \\
& \left.-\sum_{j} \int d \phi_{x} d \phi_{y} \theta\left(\phi_{y}-\phi_{x}\right)\left[M_{\mathrm{BW}, \mu}\left(\phi_{y}\right) \Lambda_{j}^{\mu}\right]\left[M_{C, \nu}\left(\phi_{x}\right) \Lambda_{j}^{\nu}\right]\right\rangle-\{f \leftrightarrow e\} .
\end{aligned}
$$

This expression is particularly suited to studying the contributions of the intermediate photon's different polarization states to the full probability. Furthermore, it should be noticed that the gauge invariance of the amplitude $S_{f i}$ does not imply that the contribution of the terms proportional to $n^{\mu} k^{\nu}$ and to $n^{\nu} k^{\mu}$ vanishes. In fact, the requirement of gauge invariance has already been exploited and is related to the intermediate photon with fourmomentum $q^{\mu}$. Indeed, one can show that if one first constructs a basis with the four quantities $q^{\mu}, n^{\mu}$ and $\Lambda_{j}^{\prime \mu}=\left(n^{\mu} a_{j}^{\nu}-n^{\nu} a_{j}^{\mu}\right) q_{\nu} / q_{-}$; separates out the transverse polarization contribution (notice that after the integrals over the transverse and the $T$ coordinates are taken, one obtains $\Lambda_{j}^{\prime \mu}=\Lambda_{j}^{\mu}$ ); and then imposes gauge invariance, one again obtains Eq. (24).

The result in Eq. (24) is our main analytical result. By introducing the reduced amplitudes for the direct and the cascade channels as

$$
\begin{aligned}
& M_{d}= i \int d \phi e^{-i\left[S_{\mathrm{BW}}(\phi)+S_{C}(\phi)\right]}\left\{\frac{2}{k_{-}} \frac{\bar{u}_{e} \hat{n} v_{p} \bar{u}_{f} \hat{n} u_{i}}{\gamma_{C}+\gamma_{\mathrm{BW}}}\left[\left(\alpha_{C}+\alpha_{\mathrm{BW}}\right) \psi(\phi)+\left(\beta_{C}+\beta_{\mathrm{BW}}\right) \psi^{2}(\phi)\right]\right. \\
&-\sum_{j} \frac{\bar{u}_{e} \hat{\Lambda}_{j} v_{p} \bar{u}_{f} \hat{\Lambda}_{j} u_{i}}{\gamma_{C} \gamma_{\mathrm{BW}}\left(\gamma_{C}+\gamma_{\mathrm{BW}}\right)}\left[\left(\alpha_{C} \gamma_{C}-\alpha_{\mathrm{BW}} \gamma_{\mathrm{BW}}\right) \psi(\phi)+\left(\beta_{C} \gamma_{C}-\beta_{\left.\left.\mathrm{BW} \gamma_{\mathrm{BW}}\right) \psi \psi^{2}(\phi)\right]}\right.\right. \\
&\left.+\sum_{j} \frac{\bar{u}_{f} \hat{\Lambda}_{j} u_{i}}{2 \gamma_{C}} \bar{u}_{e}\left[\frac{e \hat{n} \hat{A}(\phi) \hat{\Lambda}_{j}}{p_{e,-}}+\frac{e \hat{\Lambda}_{j} \hat{n} \hat{A}(\phi)}{p_{p,-}}\right] v_{p}-\frac{\bar{u}_{e} \hat{\Lambda}_{j} v_{p}}{2 \gamma_{\mathrm{BW}}} \bar{u}_{f}\left[\frac{e \hat{n} \hat{A}(\phi) \hat{\Lambda}_{j}}{p_{f,-}}-\frac{e \hat{\Lambda}_{j} \hat{n} \hat{A}(\phi)}{p_{i,-}}\right] u_{i}\right\}-\{f \leftrightarrow e\}, \\
& M_{c}=-\sum_{j} \int d \phi_{x} d \phi_{y} \theta\left(\phi_{y}-\phi_{x}\right)\left[M_{\mathrm{BW}, \mu}\left(\phi_{y}\right) \Lambda_{j}^{\mu}\right]\left[M_{C, \nu}\left(\phi_{x}\right) \Lambda_{j}^{\nu}\right]-\{f \leftrightarrow e\},
\end{aligned}
$$

we can write the differential trident probability $d P$ summed/averaged over all final/initial spin quantum numbers and integrated over the final electrons' momenta as

$$
d P=\frac{\alpha^{2} \pi^{2}}{4} \frac{1}{p_{i,-}} \frac{d^{3} \boldsymbol{p}_{p}}{(2 \pi)^{3}} \frac{1}{2 \varepsilon_{p}} \sum_{s_{i}, S_{f}, s_{e}, S_{p}} \int \frac{d^{3} \boldsymbol{p}_{e}}{(2 \pi)^{3}} \frac{1}{2 \varepsilon_{e}} \frac{1}{k_{-}^{2} p_{f,-}}\left[\left|M_{d}\right|^{2}+\left|M_{c}\right|^{2}+2 \operatorname{Re}\left(M_{d}^{*} M_{c}\right)\right]
$$

where we have exploited the three-dimensional delta function in the amplitude to take the integral in $d^{3} \boldsymbol{p}_{f}$ and where $\alpha=e^{2}$ is the fine-structure constant. This equation is equivalent to Eq. (4) of [25]. It is worth pointing out that the probability corresponding to the term $\left|M_{c}\right|^{2}$ in the integrand should not be identified yet with the cascade probability. The reason is that the quantity $\left|M_{c}\right|^{2}$ contains interference terms between different (transverse) 
polarizations of the intermediate photon and interference terms between the two amplitudes differing by the exchange of the quantum numbers of the two final electrons. In order to clearly isolate what we will call the cascade probability, which reduces to the one computed in [27-30] in the case of a constant-crossed field, we decompose the direct and the cascade amplitudes as

$$
\begin{gathered}
M_{d}=M_{d, n}^{(e f)}+M_{d, 1}^{(e f)}+M_{d, 2}^{(e f)}-M_{d, n}^{(f e)}-M_{d, 1}^{(f e)}-M_{d, 2}^{(f e)}, \\
M_{c}=M_{c, 1}^{(e f)}+M_{c, 2}^{(e f)}-M_{c, 1}^{(f e)}-M_{c, 2}^{(f e)},
\end{gathered}
$$

with the definition of each single term being clear from the expression in Eqs. (25) and (26) [for the sake of clarity we specify that the term $M_{d, n}^{(e f)}$ corresponds to the second line in Eq. (24) and that the indexes 1 and 2 refer to the different transverse polarizations of the intermediate photon]. According to this splitting of the amplitude, we write the differential probability as $d P=d P_{c}+d P_{d}+d \mathcal{P}_{i}$, where

$$
\begin{aligned}
d P_{c}= & \frac{\alpha^{2} \pi^{2}}{4} \frac{1}{p_{i,-}} \frac{d^{3} \boldsymbol{p}_{p}}{(2 \pi)^{3}} \frac{1}{2 \varepsilon_{p}} \sum_{s_{i}, s_{f}, s_{e}, s_{p}} \int \frac{d^{3} \boldsymbol{p}_{e}}{(2 \pi)^{3}} \frac{1}{2 \varepsilon_{e}} \frac{1}{k_{-}^{2} p_{f,-}} \\
& \times\left[\left|M_{c, 1}^{(e f)}\right|^{2}+\left|M_{c, 2}^{(e f)}\right|^{2}+\left|M_{c, 1}^{(f e)}\right|^{2}+\left|M_{c, 2}^{(f e)}\right|^{2}\right] \\
= & \frac{\alpha^{2} \pi^{2}}{2} \frac{1}{p_{i,-}} \frac{d^{3} \boldsymbol{p}_{p}}{(2 \pi)^{3}} \frac{1}{2 \varepsilon_{p}} \sum_{s_{i}, s_{f}, s_{e}, s_{p}} \int \frac{d^{3} \boldsymbol{p}_{e}}{(2 \pi)^{3}} \frac{1}{2 \varepsilon_{e}} \frac{1}{k_{-}^{2} p_{f,-}} \\
& \times\left[\left|M_{c, 1}^{(e f)}\right|^{2}+\left|M_{c, 2}^{(e f)}\right|^{2}\right]
\end{aligned}
$$

is the cascade-channel probability,

$$
\begin{aligned}
d P_{d}= & \frac{\alpha^{2} \pi^{2}}{4} \frac{1}{p_{i,-}} \frac{d^{3} \boldsymbol{p}_{p}}{(2 \pi)^{3}} \frac{1}{2 \varepsilon_{p}} \sum_{s_{i}, s_{f}, s_{e}, s_{p}} \int \frac{d^{3} \boldsymbol{p}_{e}}{(2 \pi)^{3}} \frac{1}{2 \varepsilon_{e}} \frac{1}{k_{-}^{2} p_{f,-}} \\
& \times\left[\left|M_{d, n}^{(e f)}\right|^{2}+\left|M_{d, 1}^{(e f)}\right|^{2}+\left|M_{d, 2}^{(e f)}\right|^{2}+\left|M_{d, n}^{(f e)}\right|^{2}\right. \\
& \left.+\left|M_{d, 1}^{(f e)}\right|^{2}+\left|M_{d, 2}^{(f e)}\right|^{2}\right] \\
= & \frac{\alpha^{2} \pi^{2}}{2} \frac{1}{p_{i,-}} \frac{d^{3} \boldsymbol{p}_{p}}{(2 \pi)^{3}} \frac{1}{2 \varepsilon_{p}} \sum_{s_{i}, s_{f}, s_{e}, S_{p}} \int \frac{d^{3} \boldsymbol{p}_{e}}{(2 \pi)^{3}} \frac{1}{2 \varepsilon_{e}} \frac{1}{k_{-}^{2} p_{f,-}} \\
& \times\left[\left|M_{d, n}^{(e f)}\right|^{2}+\left|M_{d, 1}^{(e f)}\right|^{2}+\left|M_{d, 2}^{(e f)}\right|^{2}\right]
\end{aligned}
$$

is the direct-channel probability, and $d \mathcal{P}_{i}=d P-d P_{c}-$ $d P_{d}$ is the sum of the several interference terms, which do not need to be reported here (since $d \mathcal{P}_{i}$ can be negative we have used a different symbol to indicate it). Before passing to the numerical results, we would like to show explicitly how the quantity $P_{c}=\int d P_{c}$, with the integral being meant to be over the positron momentum [see Eq. (30)], reduces to the cascade probability in the local constant field approximation (see [27-30]). Since in Eq. (30) we decided to write the probability in terms of $M_{c, j}^{(e f)}$, we consider the following one-vertex processes:

(1) Nonlinear Compton scattering by an electron with four-momentum $p_{i}^{\mu}$ and spin quantum number $s_{i}$ which emits a (real) photon with four-momentum $k^{\mu}$ and (transverse) polarization $j$ and remains with four-momentum $p_{f}^{\mu}$ and spin quantum number $s_{f}$;

(2) Nonlinear Breit-Wheeler pair production by a (real) photon with four-momentum $k^{\mu}$ and (transverse) polarization $j$, which transforms into an electron with four-momentum $p_{e}^{\mu}$ and spin quantum number $s_{e}$ and a positron with four-momentum $p_{p}^{\mu}$ and spin quantum number $s_{p}$.

The regularized probability amplitudes of these two processes can be written as

$$
\begin{aligned}
S_{C, j}= & -i e \sqrt{\frac{4 \pi}{8 \varepsilon_{i} \varepsilon_{f} \omega}}(2 \pi)^{3} \delta\left(p_{f,-}+k_{-}-p_{i,-}\right) \\
& \times \delta^{(2)}\left(\boldsymbol{p}_{f, \perp}+\boldsymbol{k}_{\perp}-\boldsymbol{p}_{i, \perp}\right) \int d \phi_{x} M_{C, \nu}\left(\phi_{x}\right) \Lambda_{j}^{\nu},
\end{aligned}
$$

$$
\begin{aligned}
S_{\mathrm{BW}, j}= & -i e \sqrt{\frac{4 \pi}{8 \varepsilon_{e} \varepsilon_{p} \omega}}(2 \pi)^{3} \delta\left(p_{e,-}+p_{p,-}-k_{-}\right) \\
& \times \delta^{(2)}\left(\boldsymbol{p}_{e, \perp}+\boldsymbol{p}_{p, \perp}-\boldsymbol{k}_{\perp}\right) \int d \phi_{y} M_{\mathrm{BW}, \nu}\left(\phi_{y}\right) \Lambda_{j}^{\nu},
\end{aligned}
$$

and we compare these amplitudes with the cascade amplitude

$$
\begin{aligned}
S_{c, j}^{(e f)}= & -e^{2} \sqrt{\frac{(4 \pi)^{2}}{64 \varepsilon_{i} \varepsilon_{f} \omega^{2} \varepsilon_{e} \varepsilon_{p}}} \frac{\omega}{k_{-}}(2 \pi)^{3} \delta\left(p_{e,-}+p_{f,-}+p_{p,-}-p_{i,-}\right) \delta^{(2)}\left(\boldsymbol{p}_{e, \perp}+\boldsymbol{p}_{f, \perp}+\boldsymbol{p}_{p, \perp}-\boldsymbol{p}_{i, \perp}\right) \\
& \times \int d \phi_{x} d \phi_{y} \theta\left(\phi_{y}-\phi_{x}\right)\left[M_{\mathrm{BW}, \mu}\left(\phi_{y}\right) \Lambda_{j}^{\mu}\right]\left[M_{C, \nu}\left(\phi_{x}\right) \Lambda_{j}^{\nu}\right]
\end{aligned}
$$

corresponding to the partial amplitude $M_{c, j}^{(e f)}$. In order to calculate the transition probabilities, we have to square the delta functions and it is convenient first to use the transformations (we have implicitly employed the last of these transformations already above when we computed the differential probability $d P$ ) 


$$
\begin{array}{r}
\delta\left(p_{f,-}+k_{-}-p_{i,-}\right)=\frac{\varepsilon_{i}}{p_{i,-}} \delta\left(p_{i, n}-\bar{p}_{i, n}\right), \\
\delta\left(p_{e,-}+p_{p,-}-k_{-}\right)=\frac{\omega}{k_{-}} \delta\left(k_{n}-\bar{k}_{n}\right), \\
\delta\left(p_{e,-}+p_{f,-}+p_{p,-}-p_{i,-}\right)=\frac{\varepsilon_{i}}{p_{i,-}} \delta\left(p_{i, n}-\bar{p}_{i, n}^{\prime}\right)
\end{array}
$$

to the corresponding longitudinal components of the momenta, where the exact expressions of the quantities $\bar{p}_{i, n}, \bar{k}_{n}$, and $\bar{p}_{i, n}^{\prime}$ are not necessary here. By computing the modulus square of the above amplitudes, we obtain the following probabilities:

$$
\begin{gathered}
\frac{d P_{C, j}}{d^{3} \boldsymbol{k}}=\frac{e^{2}}{2} \frac{1}{(2 \pi)^{3}} \sum_{s_{i}, s_{f}} \int \frac{d^{3} \boldsymbol{p}_{f}}{(2 \pi)^{3}} \frac{\omega}{k_{-}} \frac{\varepsilon_{i}}{p_{i,-}}(2 \pi)^{3} \delta^{(3)}\left(\boldsymbol{k}-\boldsymbol{k}_{0}\right)\left|\int d \phi_{x} M_{C, \nu}\left(\phi_{x}\right) \Lambda_{j}^{\nu}\right|^{2}, \\
P_{\mathrm{BW}, j}=e^{2} \sum_{s_{e}, s_{p}} \int \frac{d^{3} \boldsymbol{p}_{p}}{(2 \pi)^{3}} \frac{\omega}{k_{-}} \frac{\varepsilon_{e}}{p_{e,-}}\left|\int d \phi_{y} M_{\mathrm{BW}, \mu}\left(\phi_{y}\right) \Lambda_{j}^{\mu}\right|^{2}, \\
P_{c, j}^{(e f)}=\frac{e^{4}}{2} \sum_{s_{i}, s_{f}, s_{e}, s_{p}} \int \frac{d^{3} \boldsymbol{p}_{p}}{(2 \pi)^{3}} \int \frac{d^{3} \boldsymbol{p}_{f}}{(2 \pi)^{3}} \frac{\omega^{2}}{k_{-}^{2}} \frac{\varepsilon_{i}}{p_{i,-}} \frac{\varepsilon_{e}}{p_{e,-}}\left|\int d \phi_{x} d \phi_{y} \theta\left(\phi_{y}-\phi_{x}\right)\left[M_{\mathrm{BW}, \mu}\left(\phi_{y}\right) \Lambda_{j}^{\mu}\right]\left[M_{C, \nu}\left(\phi_{x}\right) \Lambda_{j}^{\nu}\right]\right|^{2} .
\end{gathered}
$$

Note that, in order to calculate the cascade probability out of the two probabilities of nonlinear Compton scattering and nonlinear Breit-Wheeler pair production, one needs initially only the differential probability of nonlinear Compton scattering in the photon momentum. In this respect, it was convenient to write the three-dimensional delta function in terms of the emitted photon momentum and, as above, the expression of the momentum $\boldsymbol{k}_{0}$ is not needed. Also, we observe that it makes physical sense to talk about a cascade process only when the probabilities can be expressed as integrals over laser phases (or times for external fields of different structures) of corresponding probabilities per unit phase (time), which depend only on the local value of the plane wave (external field) at that phase (time). We now first focus on the one-vertex processes and thus imagine to work in the local constant-crossed field limit where the classical nonlinearity parameter $\xi$ is very large. When we square the amplitude, e.g., of nonlinear Compton scattering, we obtain a double integral in $\phi_{x}$ and, say, $\phi_{x}^{\prime}$ [see Eq. (32)]. Since in the local constant-crossed field limit the dominant contribution to the probabilities comes from the region where the quantity $\left|\phi_{x}^{\prime}-\phi_{x}\right|$ is much smaller (by a factor of the order of $1 / \xi)$ than the laser central period [16,33], it is convenient to pass to the variables $\phi_{x,+}=\left(\phi_{x}^{\prime}+\phi_{x}\right) / 2$ and $\phi_{x,-}=\phi_{x}^{\prime}-$ $\phi_{x}$ and expand the integrand with respect to $\phi_{x,-}$. The procedure is well known (see, e.g., [34]) and it is not necessary to report the details here. It is important to point out that the probability of nonlinear Compton scattering in this limit can be written in the form $d P_{C, j} / d^{3} \boldsymbol{k}=$ $\int d \phi_{x,+} d P_{C, j}\left(\phi_{x,+}\right) / d \phi_{x,+} d^{3} \boldsymbol{k}$, where

$$
\begin{aligned}
\frac{d P_{C, j}\left(\phi_{x,+}\right)}{d \phi_{x,+} d^{3} \boldsymbol{k}}= & \frac{e^{2}}{2} \frac{1}{(2 \pi)^{3}} \sum_{s_{i}, s_{f}} \int \frac{d^{3} \boldsymbol{p}_{f}}{(2 \pi)^{3}} \frac{\omega}{k_{-}} \frac{\varepsilon_{i}}{p_{i,-}}(2 \pi)^{3} \delta^{(3)}\left(\boldsymbol{k}-\boldsymbol{k}_{0}\right) \\
& \times \int d \phi_{x,-}\left[M_{C, \nu}\left(\phi_{x,+}-\phi_{x,-} / 2\right) \Lambda_{j}^{\nu}\right]\left[M_{C, \nu^{\prime}}^{*}\left(\phi_{x,+}+\phi_{x,-} / 2\right) \Lambda_{j}^{\nu^{\prime}}\right],
\end{aligned}
$$

with $d P_{C, j}\left(\phi_{x,+}\right) / d \phi_{x,+} d^{3} \boldsymbol{k}$ being a non-negative quantity (in this limit) depending only on the plane-wave electromagnetic field calculated at $\phi_{x,+}$. Analogously, one can write in the same limit that $P_{\mathrm{BW}, j}=\int d \phi_{y,+} d P_{\mathrm{BW}, j}\left(\phi_{y,+}\right) / d \phi_{y,+}$, where

$$
\frac{d P_{\mathrm{BW}, j}\left(\phi_{y,+}\right)}{d \phi_{y,+}}=e^{2} \sum_{s_{e}, s_{p}} \int \frac{d^{3} \boldsymbol{p}_{p}}{(2 \pi)^{3}} \frac{\omega}{k_{-}} \frac{\varepsilon_{e}}{p_{e,-}} \int d \phi_{y,-}\left[M_{\mathrm{BW}, \mu}\left(\phi_{y,+}-\phi_{y,-} / 2\right) \Lambda_{j}^{\mu}\right]\left[M_{\mathrm{BW}, \mu^{\prime}}^{*}\left(\phi_{y,+}+\phi_{y,-} / 2\right) \Lambda_{j}^{\mu^{\prime}}\right],
$$

with $\phi_{y,+}=\left(\phi_{y}^{\prime}+\phi_{y}\right) / 2$ and $\phi_{y,-}=\phi_{y}^{\prime}-\phi_{y}$. We checked analytically that Eqs. (41) and (42) are equivalent to Eqs. (20) from [15], respectively, which were demonstrated to be obtainable from Eqs. (36) and (37) of [25], respectively, when considering a constant-crossed field. Now, it is clear that the total cascade probability $\tilde{P}_{c}$ calculated out of the two elementary processes of nonlinear Compton scattering and nonlinear Breit-Wheeler pair production is given by 


$$
\begin{aligned}
\tilde{P}_{c}= & \sum_{j} \int d^{3} \boldsymbol{k} \int d \phi_{x,+} d \phi_{y,+} \theta\left(\phi_{y,+}-\phi_{x,+}\right) \frac{d P_{\mathrm{BW}, j}\left(\phi_{y,+}\right)}{d \phi_{y,+}} \frac{d P_{C, j}\left(\phi_{x,+}\right)}{d \phi_{x,+} d^{3} \boldsymbol{k}} \\
= & \frac{e^{4}}{2} \sum_{j} \sum_{s_{i}, s_{e}, s_{f}, s_{p}} \int d \phi_{x,+} d \phi_{y,+} \theta\left(\phi_{y,+}-\phi_{x,+}\right) \int \frac{d^{3} \boldsymbol{p}_{p}}{(2 \pi)^{3}} \int \frac{d^{3} \boldsymbol{p}_{f}}{(2 \pi)^{3}} \frac{\omega^{2}}{k_{-}^{2}} \frac{\varepsilon_{i}}{p_{i,-}} \frac{\varepsilon_{e}}{p_{e,-}} \\
& \times \int d \phi_{y,-} d \phi_{x,-}\left[M_{\mathrm{BW}, \mu}\left(\phi_{y,+}-\phi_{y,-} / 2\right) \Lambda_{j}^{\mu}\right]\left[M_{\mathrm{BW}, \mu^{\prime}}^{*}\left(\phi_{y,+}+\phi_{y,-} / 2\right) \Lambda_{j}^{\mu^{\prime}}\right] \\
& \times\left[M_{C, \nu}\left(\phi_{x,+}-\phi_{x,-} / 2\right) \Lambda_{j}^{\nu}\right]\left[M_{C, \nu^{\prime}}^{*}\left(\phi_{x,+}+\phi_{x,-} / 2\right) \Lambda_{j}^{\nu^{\prime}}\right]
\end{aligned}
$$

which is equivalent to $\mathbb{P}_{2}$ defined in Eq. (39) of [25], confirming that the constant-crossed field limit reproduces literature results. Now, looking back at Eq. (40), imagining performing the sum over $j$, and then working in the local constant field limit, we easily realize that equation coincides with Eq. (43) if the approximation $\theta\left(\phi_{y}-\phi_{x}\right) \theta\left(\phi_{y}^{\prime}-\phi_{x}^{\prime}\right) \approx$ $\theta\left(\phi_{y,+}-\phi_{x,+}\right)$ holds in the same limit. In fact, the amplitudes of the elementary processes (nonlinear Compton scattering and nonlinear Breit-Wheeler pair production) are exactly the same [and given by Eqs. (22)-(23)] and the limiting procedure is the same for both equations. The above approximate identity between theta functions can be proved starting from the identity $\theta(a) \theta(b)=\theta(a b) \theta(a+b)$ valid for any pair of real numbers $a$ and $b$, which in our case provides the identity

$$
\begin{aligned}
& \theta\left(\phi_{y}-\phi_{x}\right) \theta\left(\phi_{y}^{\prime}-\phi_{x}^{\prime}\right) \\
& \quad=\theta\left(\phi_{y,+}-\phi_{x,+}\right) \theta\left(\left(\phi_{y}-\phi_{x}\right)\left(\phi_{y}^{\prime}-\phi_{x}^{\prime}\right)\right) .
\end{aligned}
$$

Now, we use the identity $\theta(a b) \theta(a+b)=\{1-[\theta(a)-$ $\left.\theta(b)]^{2}\right\} \theta(a+b)=[1-\theta(|a-b| / 2-(a+b) / 2)] \theta(a+b)$ to finally obtain

$$
\begin{aligned}
& \theta\left(\phi_{y}-\phi_{x}\right) \theta\left(\phi_{y}^{\prime}-\phi_{x}^{\prime}\right) \\
& \quad=\theta\left(\phi_{y,+}-\phi_{x,+}\right)\left[1-\theta\left(\frac{\left|\phi_{y,-}-\phi_{x,-}\right|}{2}-\left(\phi_{y,+}-\phi_{x,+}\right)\right)\right],
\end{aligned}
$$

which approximately turns into the needed equality once we observe that in the local constant field limit we can neglect the small quantity $\left|\phi_{y,-}-\phi_{x,-}\right| / 2$. An analogous derivation was used in [25] to relate the cascade contribution of the full trident scattering rate to a product approach of multiplying the rates of Compton scattering and Breit-Wheeler pair production on the probability level. One can alternatively define as a cascade term only the one coming from the first term in Eq. (45), which is still exact.

\section{NUMERICAL INVESTIGATIONS}

In the following we wish to exemplify the analytical finding of Eqs. (24),(27),(30),(31) in a series of numerical test cases. Before we turn to quantitative results, however, we wish to repeat that the main advantage of Eq. (24) is that in the following we will not encounter any singular phase integrals. The reason for this simplification is that the amplitude itself is already fully regularized with all singularities removed. On the contrary, the resulting probabilities do not have a Gaussian form with respect to the transverse momenta of the final particle, which, unlike in [25], prevents us from performing the corresponding integrals analytically. Now, in order to obtain these desired numerical results, the multidimensional dynamic integrals defined in Eqs. (15)(20) need to be evaluated numerically. To this end, we employ a well-established multidimensional Filon-type integration routine [32,35] (see [36] for details of this method's computational implementation). Inserting the numerical values of these integral expressions into Eq. (24) then results in a complex-valued result for the scattering matrix amplitude for each combination of the four particles' polarizations. This explicit polarization dependence makes it easily feasible to study polarization dynamics of the pair production based on Eq. (24). But as here we do not wish to elaborate on the intricacies of the polarized cross section, we numerically sum and average over the final and initial state particles' polarizations, respectively. To this end we represent the particles' two polarization states with the two standard spinors for electrons and positrons [18],

$u_{n}=\left(\begin{array}{c}\sqrt{\varepsilon+m} w_{n} \\ \frac{\sigma \cdot p}{\sqrt{\varepsilon+m}} w_{n}\end{array}\right), \quad v_{n}=\left(\begin{array}{c}\frac{\sigma \cdot p}{\sqrt{\varepsilon+m}} w_{n}^{\prime} \\ \sqrt{\varepsilon+m} w_{n}^{\prime}\end{array}\right)$,

respectively, with $n \in[1,2]$ and where $w_{n}$ and $w_{n}^{\prime}=$ $i(-1)^{n+1} w_{n}$ are arbitrary two-dimensional unit vectors and we chose $w_{n}=\left(\delta_{n 1}, \delta_{n 2}\right)$. The remaining integrals over the final particles' phase space are then also computed numerically by a Simpson rule quadrature.

Before we turn to the specific examples, however, we wish to benchmark our numerics against the known literature results of the SLAC experiment 144 on trident pair production [21,37]. In this experiment, a laser pulse with a peak nonlinearity parameter of $\xi \approx 0.5$ and wavelength $\lambda=527 \mathrm{~nm}$ was brought into collision with the SLAC beam of $46.6 \mathrm{GeV}$ electrons under a collision angle 


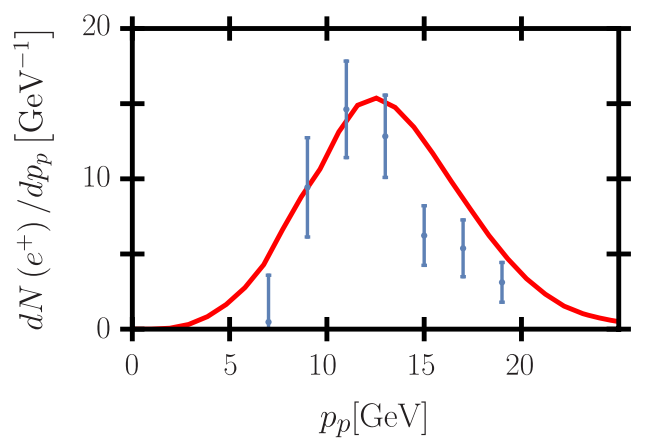

FIG. 2. Comparing our model derived by numerically evaluating Eq. (24) (solid red line) to the experimental data of [37] (blue dots with experimental error bars) by averaging over the focal volume of the used laser and multiplying with the number of electrons in each bunch and the total number of successful shots.

of $\theta_{0}=17^{\circ}$. We model this interaction geometry by calculating the positron production probability of a single electron colliding under an angle $\theta_{0}$ with a plane-wave laser pulse with a flat-top temporal profile of an effective duration of $40 \mathrm{fs}$, corresponding to the transit time of the electron through the laser focus, and one-cycle rising and falling edges, in analogy to [13]. In order to account for the transverse change of the field strength over the laser's finite focal volume, which is not included in our plane-wave model, we average our calculations over this volume by multiplying the results for varying values of $\xi$ with the focal volume's portion in which that particular value of $\xi$ is reached. We then convert our results to the units used in [37] for better comparability; i.e., we show the spectrum of produced particles in $\mathrm{GeV}^{-1}$ as a function of the produced positrons' momentum in $\mathrm{GeV}$. In order to recover the spectrum of the total number of produced positrons [37] we then have to multiply our result with the total number 21962 of used shots and the corrected estimate of $5 \times 10^{9}$ electrons per bunch [21]. Comparing the resulting prediction of our calculations to the data points of Fig. 3(b) in [37] we find good agreement (see Fig. 2). Notably, we find the spectral peak of the produced positrons located at a momentum comparable to the peak of the experimental data, in contrast to earlier predictions of harder than observed spectra [21]. Integrating the shown spectrum over momenta we obtain a positron production probability of $8 \times 10^{-3}$ per successful shot, resulting in an integrated production of $N_{e^{+}} \approx$ 176 positrons over the whole experimental run, which is in excellent agreement to the number of $175 \pm 13$ produced positrons reported in [37].

Having established this benchmark, in the following we are going to study fully differential rates. To this end, we fix the observation directions of the positron and one of the final state electrons, use energy-momentum conservation laws of the scattering amplitude to fix the second electron's momentum and skip the phase space integrations of the

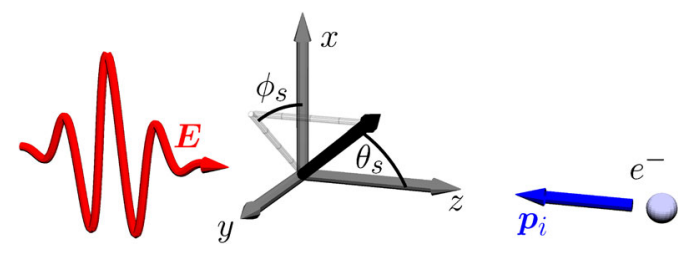

FIG. 3. Spherical coordinate frame in which we study the NTPP process with the polar and azimuthal emission angles $\left(\theta_{s}, \phi_{s}\right)$, respectively, indicated.

final particles. By varying the observation angles for the positron and final state electrons we can hence provide the first angularly resolved NTPP probabilities in a planewave laser pulse. As we are interested in deviations from the constant-crossed field limit, we consider a linearly polarized laser pulse with the standard short pulse-shape function [35]

$$
\psi(\phi)=\sin ^{4}\left(\frac{\omega_{0} \phi}{2 N}\right) \sin \left(\omega_{0} \phi\right)
$$

propagating along the $z$-axis, which we additionally assume to collide head-on with an ultrarelativistic electron (see Fig. 3). Furthermore, we assume the laser to be in the optical regime $\omega_{0}=1.55 \mathrm{eV}$ and its duration to be ultrashort, comprising only $N=2$ cycles of the carrier wave.

As we are considering a linearly polarized laser pulse, we expect most of the classical particle dynamics and positron production to occur on the plane identified by the laser propagation direction and the laser polarization direction. In a spherical coordinate frame (see Fig. 3) this plane is denoted by the $x-z$ plane, so we focus most of our discussion on observing particles in either $\phi_{s}=0$ or $\phi_{s}=\pi$. Furthermore, we note that in the regime $\varepsilon_{i}, \omega \gg m \xi$, as we study here, final state particles in nonlinear Compton scattering and NBWPP are angularly confined around the initial state electron's and photon's propagation direction, respectively, to a narrow cone of opening angle $\theta_{s} \sim m \xi / \varepsilon_{i}$ and $\theta_{s} \sim m \xi / \omega$, respectively. We thus observe the final state particles of NTPP in a direction close to the initial state electron's propagation direction, in a head-on collision along the $z$-axis given by $\theta_{s}=\pi$. Finally, in all examples we are going to compare the probabilities $P_{c}$ and $P_{d}$, derived from the direct squares of $M_{c}$ and $M_{d}$, defined in Eq. (30) and Eq. (31), respectively, to the full probability defined in Eq. (27). Consequently, in contrast to $[25,26]$ we do not explicitly consider the interference term of the pair production probability separately.

We begin by studying a case typical of nowadays feasible all-optical experiments in which an electron of initial energy $\varepsilon_{i}=1 \mathrm{GeV}$ collides with a laser pulse of intensity $I=2 \times 10^{21} \mathrm{~W} / \mathrm{cm}^{2}(\xi \approx 22)$, yielding a comparatively small quantum nonlinearity parameter $\chi \approx 0.25$. We find the full pair production probability to be completely dominated by the cascade process [see Figs. 4(b) and 4(c)], in agreement with earlier studies in the regime $\xi \gg 1, \chi \ll 1$ [25], with the direct contribution suppressed 


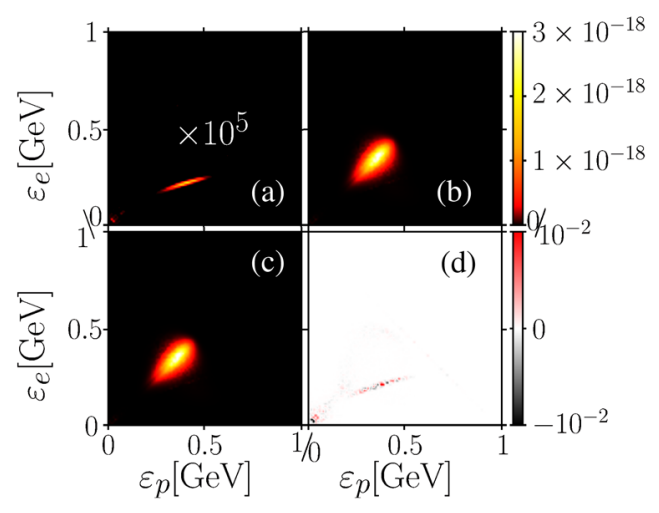

FIG. 4. Differential NTPP probability of direct (a), cascade (b) and full (c) channels for the collision of an electron with initial energy $\varepsilon_{i}=1 \mathrm{GeV}$ with a laser pulse of intensity $I=$ $2 \times 10^{21} \mathrm{~W} / \mathrm{cm}^{2}(\xi \approx 22, \chi \approx 0.25)$; the positron observed at $\left(\theta_{s}, \phi_{s}\right)=\left(\pi-m \xi / \varepsilon_{i}, \pi / 2\right)$; and one of the electrons at $\left(\theta_{s}, \phi_{s}\right)=\left(\pi-m \xi / \varepsilon_{i}, 0\right)$. The relative error of the cascade approximation $\mathcal{R}$ is shown in (d).

by about 5 orders of magnitude [see Fig. 4(a)]. The dominance of the cascade contribution is even more obvious from studying the relative error made by approximating the full NTPP probability with the cascade contribution, distinguished by the parameter

$$
\mathcal{R}=\frac{d P-d P_{c}}{d P} .
$$

In the current case we find this parameter to be of percent level in the particle energy regime where the direct channel is strongest. However, we note that the deviation is not a pure contribution of the direct channel, but an interference effect. Furthermore, we find the probability of NTPP to be centered around the symmetry axis of the

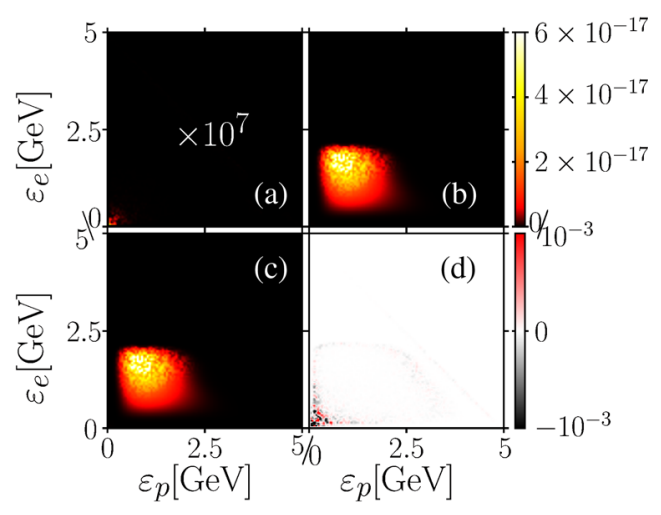

FIG. 5. Differential NTPP probability of direct (a), cascade (b) and full (c) channels for the collision of an electron with initial energy $\varepsilon_{i}=5 \mathrm{GeV}$ with a laser pulse of intensity $I=10^{22} \mathrm{~W} / \mathrm{cm}^{2}(\xi \approx 50, \chi \approx 3)$; the positron observed at $\left(\theta_{s}, \phi_{s}\right)=\left(\pi-m \xi / \varepsilon_{i}, \pi / 2\right)$; and one of the electrons at $\left(\theta_{s}, \phi_{s}\right)=\left(\pi-m \xi / \varepsilon_{i}, 0\right)$. The relative error of the cascade approximation $\mathcal{R}$ is shown in (d). energy distribution, indicating that all three final state particles share a comparable amount of energy.

We continue by studying a case deeper in the nonlinear quantum regime in which an electron of initial energy $\varepsilon_{i}=5 \mathrm{GeV}$ collides with a laser pulse of intensity $I=$ $10^{22} \mathrm{~W} / \mathrm{cm}^{2}(\xi \approx 50)$, yielding a larger quantum nonlinearity parameter $\chi \approx 3$, likely to be close to the optimum operation parameters for upcoming laser facilities. Due to the increased laser intensity, we find the full pair production probability to be even more strongly dominated by the cascade process [see Figs. 5(b) and 5(c)], with the direct contribution's suppression increased to 7 orders of magnitude [see Fig. 5(a)] and contributing only at the smallest final state particle energies. The relative error of the cascade approximation is consequently found to be most significant at small final state particle energies, where the direct channel is strongest, but to be overall small on the level of a per mill [see Fig. 5(d)].

These findings further corroborate the common approximation of higher order nonlinear QED effects, notably NTPP, by their cascade contributions [22,23]. In order to explore the limitations of this approximation, we turn to a parameter regime where its applicability is expected to be less justified. We study a regime with a high quantum nonlinearity parameter but relatively small laser intensity $[15,26]$. Consequently, we consider the initial electron to have a very high energy of $\varepsilon_{i}=100 \mathrm{GeV}$. In combination with a laser intensity of $I=2 \times 10^{21} \mathrm{~W} / \mathrm{cm}^{2}(\xi \approx 22)$ this results in a quantum nonlinearity parameter of $\chi \approx 26$. Analyzing now the direct contribution on the same scale as the cascade and full contributions, we find its impact to be no longer negligible at low particle energies (see Fig. 6). Furthermore, we find larger positron than electron energies to be favored in this regime, as apparent from the asymmetric distributions of the energy spectra

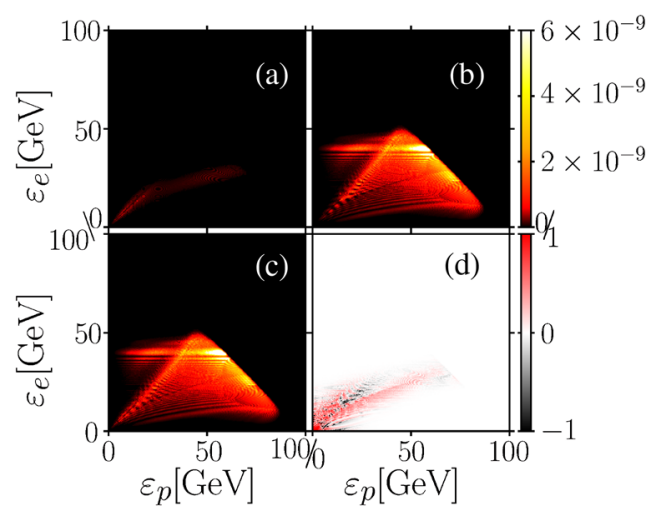

FIG. 6. Differential NTPP probability of direct (a), cascade (b) and full (c) channels for the collision of an electron with initial energy $\varepsilon_{i}=100 \mathrm{GeV}$ with a laser pulse of intensity $I=$ $2 \times 10^{21} \mathrm{~W} / \mathrm{cm}^{2} \quad(\xi \approx 22, \chi \approx 26)$; the positron observed at $\left(\theta_{s}, \phi_{s}\right)=\left(\pi-m \xi / \varepsilon_{i}, \pi\right)$; and one of the electrons at $\left(\theta_{s}, \phi_{s}\right)=\left(\pi-m \xi / \varepsilon_{i}, 0\right)$. The relative error of the cascade approximation $\mathcal{R}$ is shown in (d). 


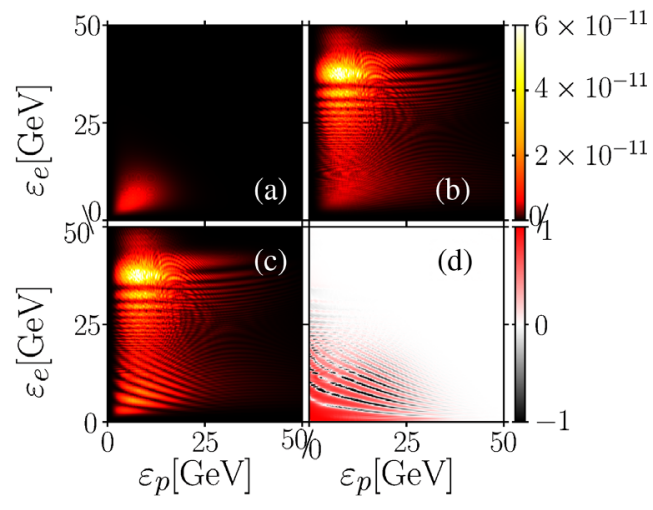

FIG. 7. Differential NTPP probability of direct (a), cascade (b) and full (c) channels for the collision of an electron with initial energy $\varepsilon_{i}=100 \mathrm{GeV}$ with a laser pulse of intensity $I=$ $5 \times 10^{20} \mathrm{~W} / \mathrm{cm}^{2}(\xi \approx 11, \chi \approx 13)$; the positron observed at $\left(\theta_{s}, \phi_{s}\right)=\left(\pi-m \xi / \varepsilon_{i}, \pi / 2\right)$; and one of the electrons at $\left(\theta_{s}, \phi_{s}\right)=\left(\pi-m \xi / \varepsilon_{i}, 0\right)$. The relative error of the cascade approximation $\mathcal{R}$ is shown in (d).

[see Figs. 6(a)-6(c)]. Interestingly, in the cascade contribution we also find considerable interference fringes, depending almost exclusively on the electron's energy [see Fig. 6(b)]. This is most probably due to the fact that the final state contains two indistinguishable electrons whose distributions can interfere. The relative error of the cascade approximation, on the other hand, is found to reach the level of $100 \%$ for small final state particle energies [see Fig. 6(d)], indicating that at these extreme parameters the cascade approximation starts to lose applicability.

We can even further enhance the visibility of the direct channel by considering a lower laser intensity. Furthermore, as a semiclassical picture of NTPP predicts the particle production to be mostly confined to the laser's plane of polarization, we can expect to observe stronger deviations from the cascade model, by observing one of the electrons inside the polarization plane $\left(\theta_{s}, \phi_{s}\right)=$ $\left(\pi-m \xi / \varepsilon_{i}, 0\right)$ but the positron in a direction perpendicular to this plane $\left(\theta_{s}, \phi_{s}\right)=\left(\pi-m \xi / \varepsilon_{i}, \pi / 2\right)$. We note, however, that for smaller initial electron energies observing the positron perpendicularly to the laser's plane of polarization does not result in a significant contribution from the direct channel (see Fig. 4). For a large initial electron energy of $\varepsilon_{i}=100 \mathrm{GeV}$, on the other hand, we indeed find that in the collision with a laser pulse of intensity $I=5 \times$ $10^{20} \mathrm{~W} / \mathrm{cm}^{2}(\xi \approx 11, \chi \approx 13)$, the direct contribution is more pronounced in comparison to the cascade channel [see Fig. 7(a)]. Again, we find the cascade channel's interference fringes to depend dominantly on the electron's energy [see Fig. 7(b)]. In the full NTPP probability, however, at small final state particle energies we find the interference fringes to exhibit a dependence on the positron's energy as well. This is a clear indication that the direct channel and interference terms between exchange diagrams start to affect the full NTPP rate [see Fig. 7(c)]. Furthermore, we find the full NTPP signal to be significantly enhanced at low particle energies, as is also apparent from the relative error $\mathcal{R}$, which is significant for low particle energies [see Fig. 7(d)].

\section{CONCLUSIONS}

We have further advanced the theoretical understanding of two aspects of NTPP. Specifically, we have found a regularized expression of the amplitude of NTPP, which exploits the gauge invariance of QED, and which allows a relatively easy numerical computation of the probability of NTPP. The regularized amplitude is naturally split into a cascade and direct contribution. Moreover, we have for the first time provided angularly resolved information about nonlinear trident pair production in a pulsed plane wave. We confirmed the cascade probability to reduce to the common product of nonlinear Compton scattering and Breit-Wheeler pair production probabilities in the case of a constantcrossed field, in agreement with other recent studies, and isolated the contributions of noncascade parts to NTPP. By squaring the amplitudes we found the observable probabilities for NTPP via the cascade and direct channels and analyzed the latter in exemplary cases. Our numerical analyses further confirmed the applicability of the cascade approximation of NTPP at low initial electron energies and high laser intensities $(\xi \gg 1, \chi \sim 1)$, but also indicated that at very high initial electron energies (such that $\chi \gg 1$ ), noncascade contributions may affect the full NTPP rate.

\section{ACKNOWLEDGMENTS}

The authors acknowledge fruitful discussions with C. H. Keitel, B. King and C. Müller.
[1] V. Yanovsky, V. Chvykov, G. Kalinchenko, P. Rousseau, T. Planchon, T. Matsuoka, A. Maksimchuk, J. Nees, G. Cheriaux, G. Mourou, and K. Krushelnick, Opt. Express 16, 2109 (2008).
[2] C. Danson et al., Nucl. Fusion 44, S239 (2004).

[3] Extreme Light Infrastructure-Whitebook, edited by G. A. Mourou, G. Korn, W. Sandner, and J. L. Collier (Andreas Thoss, Berlin, 2011). 
[4] XCELS, http://www.xcels.iapras.ru/.

[5] T. Nousch, D. Seipt, B. Kämpfer, and A. Titov, Phys. Lett. B 715, 246 (2012).

[6] K. Krajewska, C. Müller, and J. Z. Kamiński, Phys. Rev. A 87, 062107 (2013).

[7] A. Gonoskov, I. Gonoskov, C. Harvey, A. Ilderton, A. Kim, M. Marklund, G. Mourou, and A. Sergeev, Phys. Rev. Lett. 111, 060404 (2013).

[8] M. J. A. Jansen and C. Müller, Phys. Rev. A 88, 052125 (2013).

[9] A. I. Titov, B. Kämpfer, H. Takabe, and A. Hosaka, Phys. Rev. A 87, 042106 (2013).

[10] S. Augustin and C. Müller, Phys. Lett. B 737, 114 (2014).

[11] S. Meuren, C. H. Keitel, and A. Di Piazza, Phys. Rev. D 93, 085028 (2016).

[12] A. Di Piazza, C. Mueller, K. Z. Hatsagortsyan, and C. H. Keitel, Rev. Mod. Phys. 84, 1177 (2012).

[13] H. Hu, C. Müller, and C. H. Keitel, Phys. Rev. Lett. 105, 080401 (2010).

[14] A. Ilderton, Phys. Rev. Lett. 106, 020404 (2011).

[15] B. King and H. Ruhl, Phys. Rev. D 88, 013005 (2013).

[16] V. Ritus, J. Russ. Laser Res. 6, 497 (1985).

[17] W. H. Furry, Phys. Rev. 81, 115 (1951).

[18] V. B. Berstetskii, E. M. Lifshitz, and L. P. Pitaevskii, Quantum Electrodynamics (Elsevier Butterworth-Heinemann, Oxford, 1982).

[19] W. P. Leemans, A. J. Gonsalves, H.-S. Mao, K. Nakamura, C. Benedetti, C. B. Schroeder, C. Tóth, J. Daniels, D. E. Mittelberger, S. S. Bulanov, J.-L. Vay, C. G. R. Geddes, and E. Esarey, Phys. Rev. Lett. 113, 245002 (2014).

[20] C. Bula, K. T. McDonald, E. J. Prebys, C. Bamber, S. Boege, T. Kotseroglou, A. C. Melissinos, D. D. Meyerhofer, W. Ragg, D. L. Burke, R. C. Field, G. Horton-Smith, A. C. Odian, J. E. Spencer, D. Walz, S. C. Berridge, W. M. Bugg, K. Shmakov, and A. W. Weidemann, Phys. Rev. Lett. 76, 3116 (1996).

[21] C. Bamber, S. J. Boege, T. Koffas, T. Kotseroglou, A. C. Melissinos, D. D. Meyerhofer, D. A. Reis, W. Ragg, C. Bula, K. T. McDonald, E. J. Prebys, D. L. Burke, R. C.
Field, G. Horton-Smith, J. E. Spencer, D. Walz, S. C. Berridge, W. M. Bugg, K. Shmakov, and A. W. Weidemann, Phys. Rev. D 60, 092004 (1999).

[22] A. Gonoskov, S. Bastrakov, E. Efimenko, A. Ilderton, M. Marklund, I. Meyerov, A. Muraviev, A. Sergeev, I. Surmin, and E. Wallin, Phys. Rev. E 92, 023305 (2015).

[23] C. Ridgers, J. Kirk, R. Duclous, T. Blackburn, C. Brady, K. Bennett, T. Arber, and A. Bell, J. Comput. Phys. 260, 273 (2014).

[24] T. Erber, Rev. Mod. Phys. 38, 626 (1966).

[25] V. Dinu and G. Torgrimsson, Phys. Rev. D 97, 036021 (2018).

[26] B. King and A. M. Fedotov, Phys. Rev. D 98, 016005 (2018).

[27] V. Baier, V. Strakhovenko, and V. Katkov, Sov. J. Nucl. Phys. 14, 572 (1972).

[28] V. Ritus, Nucl. Phys. B44, 236 (1972).

[29] D. Morozov and V. Ritus, Nucl. Phys. B86, 309 (1975).

[30] V. Baier, V. Katkov, and V. Strakhovenko, Sov. J. Nucl. Phys. 53, 632 (1991).

[31] D. Seipt and B. Kämpfer, Phys. Rev. D 85, 101701 (2012).

[32] F. Mackenroth and A. Di Piazza, Phys. Rev. Lett. 110, 070402 (2013).

[33] V. N. Baier, V. M. Katkov, and V. M. Strakhovenko, Electromagnetic Processes at High Energies in Oriented Single Crystals (World Scientific, Singapore, 1998).

[34] A. Di Piazza, Phys. Rev. A 95, 032121 (2017).

[35] F. Mackenroth, A. Di Piazza, and C. H. Keitel, Phys. Rev. Lett. 105, 063903 (2010).

[36] K. F. Mackenroth, Quantum Radiation in Ultra-Intense Laser Pulses (Springer Theses) (Springer, Heidelberg, 2014).

[37] D. L. Burke, R. C. Field, G. Horton-Smith, J. E. Spencer, D. Walz, S. C. Berridge, W. M. Bugg, K. Shmakov, A. W. Weidemann, C. Bula, K. T. McDonald, E. J. Prebys, C. Bamber, S. J. Boege, T. Koffas, T. Kotseroglou, A. C. Melissinos, D. D. Meyerhofer, D. A. Reis, and W. Ragg, Phys. Rev. Lett. 79, 1626 (1997). 